\title{
Nonlinear dynamics of gas bubbles in viscoelastic media
}

\author{
Xinmai Yang and Charles C. Church \\ National Center for Physical Acoustics, The University of Mississippi, University, Mississippi 38677 \\ xmyang@olemiss.edu
}

\begin{abstract}
Understanding the behavior of cavitation bubbles driven by ultrasonic fields is an important problem in biomedical acoustics. The KellerMiksis equation for nonlinear bubble dynamics is combined with the Voigt model for viscoelastic media. Using experimentally determined values, the effects of elasticity on bubble oscillations are studied. Inertial cavitation thresholds are determined using $R_{\max } / R_{0}=2$, and subharmonic emissions are also estimated. The elasticity increases the threshold pressure for inertial cavitation, and subharmonic signals are significant only in a certain region of radii and driving pressures at a given frequency. These results should prove useful in cavitation detection and bubble-enhanced imaging work.

(C) 2005 Acoustical Society of America

PACS numbers: 43.35. Wa, 43.80.Sh, 43.35.Ei

Date Received: December 15, 2004 Date Accepted: April 11, 2005
\end{abstract}

\section{Introduction}

Bubble dynamics models are well established for bubbles in water or simple Newtonian fluids. With the development of new materials and new techniques, the study of bubble dynamics in viscoelastic media becomes necessary. The increasing importance of cavitation studies is partly due to applications in medical ultrasound. For example, the use of bubble-based contrast agents in diagnostic ultrasound has significantly increased the quality of imaging. In these situations, the media often exhibit non-Newtonian behavior. Recently, this issue has become more important due to the development of high-intensity focused ultrasound for therapeutic medicine. High-intensity sound may induce cavitation in soft tissue, and these microbubbles can have a huge impact on the distribution of the ultrasound energy. Understanding the behavior of this cavitation can provide a powerful tool for improving the quality of results of medical ultrasound in clinics.

The study of these microbubbles involves bubble oscillations in viscoelastic media. Many researchers have extended the study of bubble dynamics in Newtonian fluids to viscoelastic fluids. ${ }^{1-7}$ For the purpose of diagnostic ultrasound, relatively low-intensity sound is used. At these levels, essentially no cavitation occurs in soft tissue directly, ${ }^{8}$ and the only source of microbubbles is by injection of bubble-based contrast agents. However, the use of highintensity ultrasound will cause cavitation in soft tissue directly. These bubbles differ from contrast agent bubbles in that they (1) are free bubbles; and (2) may oscillate nonlinearly because of the high intensity of the sound field.

Previous models for bubbles in viscoelastic media were based on the Rayleigh-Plesset equation, which is not entirely appropriate for large-amplitude oscillations. We seek a model capable of accounting for large-amplitude bubble oscillations while incorporating an appropriate viscoelastic model. This will allow us to use the limited data available on the viscoelastic properties of soft tissue at megahertz frequencies. The Keller-Miksis equation has been shown to be suitable for large-amplitude bubble oscillations. ${ }^{9,10}$ In this study, we combine the general form of the Keller-Miksis equation with the Voigt model for viscoelasticity.

The importance of the inertial cavitation threshold has been addressed by many authors. In a medical context, severe bioeffects, including both thermal and nonthermal effects, may be induced or exacerbated by inertial cavitation during high-intensity ultrasound insonations. When inertial cavitation occurs, strong nonlinear acoustic emissions can be 
detected. A sudden change in the emission signals from a bubble is often used to monitor the occurrence of inertial cavitation in experiments. ${ }^{11}$

In addition to the familiar second, third, and higher harmonics, bubbles will also generate subharmonic signals when they oscillate nonlinearly. Therefore, monitoring the generation of subharmonics also can be used to detect inertial cavitation. Higher frequency emissions are very easily attenuated, and the signal that needs to be detected may become very weak. At the same time, the nonlinear propagation of ultrasound will also generate higher harmonics, and this can be a noise source for higher harmonic detection. Compared to harmonic detectors, subharmonic detectors have the advantages that the low-frequency signal is less attenuated in soft tissues, and bubbles are the only sources generating subharmonics in soft tissue.

Subharmonic signals have been seen in experiments during cavitation events, but mechanisms for the generation of subharmonics are still not entirely clear. Possible explanations include that a single bubble will emit subharmonics when it breaks up, or interactions inside a bubble cloud can emit subharmonics, or chaotic oscillations of a single bubble will generate subharmonics. In this study, we focus on single-bubble dynamics, and simply predict subharmonic signals from the chaotic oscillation of a single bubble. The effect of elasticity on inertial cavitation thresholds and subharmonic emissions will be investigated, providing data that may be useful for cavitation detection and bubble imaging.

\section{Theory and method}

The Keller-Miksis equation ${ }^{9,10}$ can be written in its general form as

$$
\left(1-\frac{\dot{R}}{c}\right) R \ddot{R}+\frac{3}{2}\left(1-\frac{\dot{R}}{3 c}\right) \dot{R}^{2}=\left(1+\frac{\dot{R}}{c}\right) \frac{p_{a}-p_{I}}{\rho}+\frac{R}{\rho c} \frac{d}{d t}\left[p_{a}-p_{I}\right],
$$

with

$$
p_{a}-p_{I}=p_{g}-\frac{2 \sigma}{R}-p_{0}+P_{A} \sin (\omega t)+3 \int_{R}^{\infty} \frac{\tau_{r r}}{r} d r
$$

where $p_{a}$ is the pressure at the bubble surface, $p_{I}$ is the pressure at infinity, $R$ is the bubble radius, $\dot{R}$ is the bubble wall velocity, $\ddot{R}$ is the bubble wall acceleration, $p_{g}$ is the gas pressure inside the bubble, $\sigma$ is the surface tension, $P_{A}$ is the amplitude of driving pressure, $p_{0}$ is the ambient pressure, $\tau_{r r}$ is the shear stress, $r$ is the radial direction, $c$ is the sound speed in the surrounding medium, and $\rho$ is the density of surrounding medium. This equation can account for the compressibility of the surrounding medium to the first order, and it is more suitable than the Rayleigh-Plesset equation for simulation of large amplitude of bubble oscillations. ${ }^{10}$ The validity of this equation is limited to small Mach numbers.

Since soft tissue is viscoelastic material, we need to choose a suitable viscoelastic model to determine stresses. We choose the Voigt model in this study because (1) it is a simple linear model; (2) previous work has shown that it is appropriate in the low-megahertz frequency range; and most importantly (3) some data for soft tissue are available for it. ${ }^{12,3}$ This choice also will allow us to compare our predictions with experimental measurements.

We set $\tau_{r r}=2\left(G \gamma_{r r}+\mu \dot{\gamma}_{r r}\right)$, where $\gamma_{r r}$ is the strain, $\dot{\gamma}_{r r}$ is the strain rate with $\dot{\gamma}_{r r}=\partial u / \partial r, u$ is the velocity, and $G$ is the shear modulus (or rigidity). In near field (near the bubble surface), an incompressible material assumption can be applied; thus, $u=\left(R^{2} / r^{2}\right) \dot{R}$. The gas inside the bubble is assumed ideal, and the pressure is estimated by use of a polytropic relation, $p_{g}$ $=p_{g 0}\left(R_{0} / R\right)^{3 \kappa}$, where $p_{g 0}$ is gas pressure in side a bubble at equilibrium, $R_{0}$ is the bubble equilibrium radius, and $\kappa$ is the polytropic index.

The resulting equation must be solved numerically for nonlinear oscillations. The following material properties are used in the simulation: $p_{0}=1.01 \times 10^{5} \mathrm{~Pa}, \rho=1000 \mathrm{~kg} / \mathrm{m}^{3}, c$ 




Fig. 1. A comparison of radial responses of $1-\mu \mathrm{m}$ bubbles driven by a 1-MPa, 1-MHz pulse for $G=0$ (blue line), and $G=1.0 \mathrm{MPa}$ (red line). The viscosity is fixed at $0.015 \mathrm{~Pa}$.

$=1500 \mathrm{~m} / \mathrm{s}$, and $\sigma=0.0725 \mathrm{~N} / \mathrm{m}$. All these parameters are chosen to be close to the properties of water. The real properties in soft tissue will be slightly different from these values, but with the exception of $\sigma$, the differences generally will be small. The polytropic index $\kappa=1.4$, and the elasticity and the viscosity are taken as $G=0,0.5,1.0$, or $1.5 \mathrm{MPa}$ and $\mu=0.005$ (blood) or 0.015 (tissue) Pa s. These values are chosen based on previous measurements, ${ }^{12,13}$ and they cover the experimental range of the properties of soft tissue. The effects of different viscosities in a Newtonian medium have been investigated previously. ${ }^{14,15} \mathrm{We}$ will focus on the effects of elasticity on bubble motion in this study. The chosen range of parameter values will allow us to compare results from different media. The driving frequencies used are 1 and $3.5 \mathrm{MHz}$, which are frequently used in HIFU. All the bubble oscillations are solved for 30-cycle exposures.

Several theoretical criteria for the inertial cavitation threshold have appeared in the literature, such as $T_{\max }=5000 \mathrm{~K}, R_{\max } / R_{0}=2$, etc. The different criteria will often result in different threshold values. We use $R_{\max } / R_{0}=2$ as the threshold criterion, which generally produces the lowest threshold for air bubbles in water. The strength of the subharmonic emission from a single bubble is determined relative to the amplitude of the strongest frequency component, reflecting signal-to-noise considerations in experimental detection schemes. The relative amplitude is obtained by the following method: first, the $R-t$ curve is obtained, next the radiated pressure is calculated from $p_{\mathrm{sac}}(r, t)=(\rho R / r)\left(2 \dot{R}^{2}+R \ddot{R}\right)$, where $r$ is assumed to be a unit constant, and then frequency components are determined by FFT. The amplitude of the subharmonic is expressed in decibels relative to the maximum amplitude over all frequency components.

\section{Results}

\subsection{The effect of elasticity on $R-t$ curves}

A representative example of the effect of elasticity on bubble response is shown in Fig. 1 for a $1-\mu \mathrm{m}$ bubble oscillating under a driving pressure of $1 \mathrm{MPa}$ at a frequency of $1 \mathrm{MHz}$. The viscosity is fixed at $0.015 \mathrm{~Pa}$ s, and the elasticity is chosen as either 0.0 or $1.0 \mathrm{MPa}$. As the curves in Fig. 1 show, not only is the amplitude of the oscillation much smaller when elasticity is included, the presence of the elasticity also greatly reduces the nonlinearity of the oscillation. The effect of elasticity is similarly dramatic for most parameter combinations studied. 

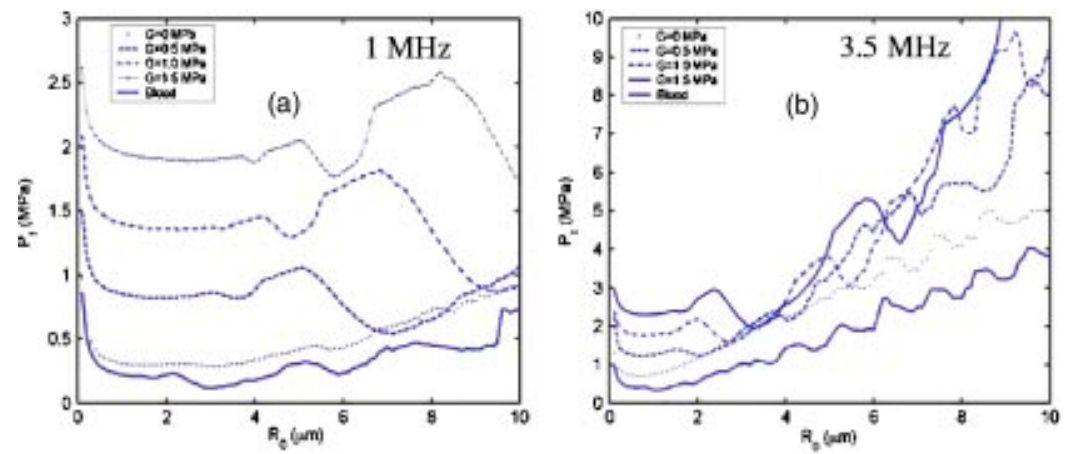

Fig. 2. Predicted thresholds for inertial cavitation at (a) $1 \mathrm{MHz}$, and (b) $3.5 \mathrm{MHz}$.

\subsection{The effect of elasticity on the inertial cavitation threshold}

In Fig. 2(a), predicted thresholds are shown for a driving frequency of $1 \mathrm{MHz}$, elasticity $G=0$, $0.5,1.0$, and $1.5 \mathrm{MPa}$ and viscosity $\mu=0.015 \mathrm{~Pa} \mathrm{~s}$. The threshold in blood, $\mu=0.005 \mathrm{~Pa}$ s, is shown for reference. The figure shows that thresholds generally increase as the elasticity increases. For radii greater than the linear resonance, the threshold curves exhibit structure that appears to be related to the fractional-order (sub)harmonic resonance minima described previously. ${ }^{16}$ The positions of the minima appear to be determined by the elasticity of the surrounding medium.

In Fig. 2(b), inertial cavitation thresholds at a driving frequency of $3.5 \mathrm{MHz}$ are shown. At this higher frequency, the increase in thresholds at larger bubble radii is much faster than that at $1 \mathrm{MHz}$. The same resonance structures can be observed on these threshold curves, the only difference being that the successive local minima are higher than their predecessors rather than lower as at $1 \mathrm{MHz}$. Overall, the threshold values are greater than those at $1 \mathrm{MHz}$.

The effect of viscosity on thresholds can be observed by comparing the curves for " $G=0 \mathrm{MPa}$ " and "blood" (the only difference between these two is the value of viscosity). This shows that the threshold increases with viscosity, and the curves have less structure at higher viscosities. In some previous measurements, ${ }^{17}$ the measured threshold values are higher than predicted in the absence of elasticity. Those measurement results may be explained by these simulations.

\subsection{The strength of subharmonic emissions}

Figure 3 shows subharmonic emissions at a frequency of $1 \mathrm{MHz}$ in a parameter space of driving pressures and bubble equilibrium radii. Results for $G=0$ and $1.0 \mathrm{MPa}$ with $\mu=0.015 \mathrm{~Pa}$ s are shown in Figs. 3(a) and (b), respectively; the result for blood is shown for reference ( $G=0$ and $\mu=0.005$ Pa s) in Fig. 3(c). We will define a subharmonic signal as "strong" when it is greater than $-30 \mathrm{~dB}$ because, above this level, the subharmonic signal can be easily detected by an 8-bit instrument. As elasticity increases, the strong subharmonic signal region moves towards larger bubble radii and higher driving pressures. Comparison with Fig. 2(a) shows that the strong subharmonic signal region generally lies above the corresponding inertial cavitation threshold. Specifically, the inertial threshold coincides with the strong subharmonic emission region better for lower values of elasticity than for higher values. This result is expected because the subharmonic signal comes from the chaotic oscillation of a bubble, and chaotic oscillations usually occur after bubble motion becomes strongly nonlinear. In comparing the map for blood [Fig. 3(c)] and that for $G=0 \mathrm{MPa}$ [Fig. 3(a)], we find that the strong subharmonic signal region becomes smaller when the viscosity increases.

Figure 3(d) shows the same result as that in Fig. 3(b) for $G=1.0 \mathrm{MPa}$, but at a driving frequency of $3.5 \mathrm{MHz}$. Compared to the results at $1 \mathrm{MHz}$, the strong subharmonic emission 

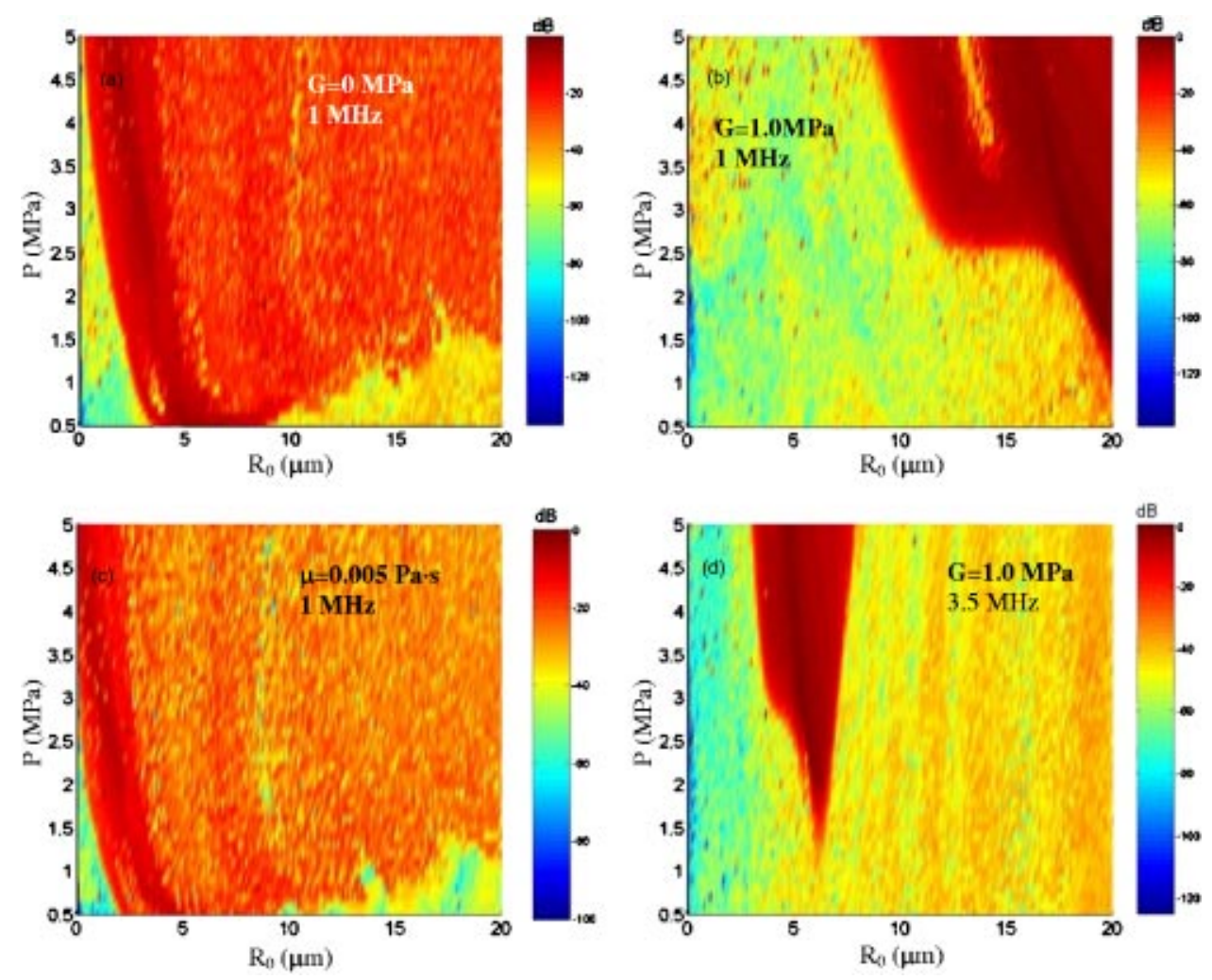

Fig. 3. Subharmonic emissions at a driving frequency of $1 \mathrm{MHz}$ in a parameter space of driving pressure and bubble equilibrium radius for (a) $G=0$ and $\mu=0.015 \mathrm{~Pa} \mathrm{~s}$; (b) $G=1.0$ and $\mu$ $=0.015 \mathrm{Pas}$; (c) blood, $G=0$ and $\mu=0.005 \mathrm{~Pa} \mathrm{~s}$; and (d) at $3.5 \mathrm{MHz}$ for $G=1.0$ and $\mu$ $=0.015 \mathrm{~Pa} \mathrm{~s}$.

region at $3.5 \mathrm{MHz}$ is much smaller and is limited to the small bubble region. This limitation to the small bubble region probably is related to the higher harmonic resonance structure of these bubbles. At $3.5 \mathrm{MHz}$, the bubble resonance size is smaller than that at $1 \mathrm{MHz}$ (3.07 versus 10.67 $\mu \mathrm{m})$. Again, all regions are above the inertial cavitation thresholds for the corresponding elasticity.

\section{Discussion and conclusions}

In this paper, the Keller-Miksis equation for nonlinear bubble dynamics was combined with the linear Voigt model for viscoelasticity. The Voigt model has the advantages of being simple and having experimental data available for use in predicting bubble responses in tissue. At high intensities, bubble oscillations are strongly nonlinear. This does not necessarily imply that a nonlinear viscoelastic model is necessary to describe the bubble motion, but the suitability of the linear model also remains unclear. Different viscoelastic models certainly will produce different predictions. The scarce measurement data at megahertz frequencies limit the study of "correct" viscoelastic models. However, the present study yields predictions, and these can be easily examined experimentally. Therefore, in future work, experiments will be conducted to verify this model. Once verified, the model also could be used to determine values for the properties of other materials.

\section{Acknowledgments}

The suggestions and support of the Biomedical Acoustic group at NCPA are gratefully acknowledged. This work was supported by award number DAMD17-02-2-0014, administered 
by the U.S. Army Medical Research Acquisition activity, Fort Detrick, MD. The information contained herein does not necessarily reflect the position or policy of the U.S. government, and no official endorsement should be inferred.

\section{References and links}

${ }^{1}$ H. S. Fogler and J. D. Goddard, "Collapse of spherical cavities in viscoelastic fluids," Phys. Fluids 13(5), 11351141 (1970).

${ }^{2}$ I. Tanasawa and W. J. Yang, "Dynamic behavior of a gas bubble in viscoelastic liquids,” J. Appl. Phys. 41(1), 4526-4531 (1970).

${ }^{3}$ A. Shima, T. Tsujino, and H. Nanjo, "Nonlinear oscillations of gas bubbles in viscoelastic fluids," Ultrasonics 24, 142-147 (1986).

${ }^{4}$ C. Kim, "Collapse of spherical bubbles in Maxwell fluids," J. Non-Newtonian Fluid Mech. 55, 33-58 (1994).

${ }^{5}$ V. N. Alekseev and S. A. Rybak, "The behavior of gas bubbles in insonated biological tissues," Acoust. Phys. 44(3), 243-247 (1998).

${ }^{6}$ J. S. Allen and R. A. Roy, "Dynamics of gas bubbles in viscoelastic fluids. I. Linear viscoelasticity," J. Acoust. Soc. Am. 107, 3167-3178 (2000).

${ }^{7}$ J. S. Allen and R. A. Roy, "Dynamics of gas bubbles in viscoelastic fluids. II. Nonlinear viscoelasticity," J. Acoust. Soc. Am. 108, 1640-1650 (2000).

${ }^{8}$ C. C. Church, "Spontaneous homogeneous nucleation, inertial cavitation and the safety of diagnostic ultrasound," Ultrasound Med. Biol. 28(10), 1349-1364 (2002).

${ }^{9}$ J. B. Keller and M. J. Miksis, "Bubble oscillations of large amplitude,” J. Acoust. Soc. Am. 68, 628-633 (1980).

${ }^{10}$ A. Prosperetti and A. Lezzi, "Bubble dynamics in a compressible liquid. First order theory," J. Fluid Mech. 168, 457-478 (1986).

${ }^{11}$ ANSI Technical Report, Bubble Detection and Cavitation Monitoring (American National Standards Institute, New York, 2002), Document ANSI S1.24 TR-2002.

${ }^{12}$ L. A. Frizzell, E. L. Carstensen, and J. F. Dyro, "Shear properties of mammalian tissues at low megahertz frequencies,” J. Acoust. Soc. Am. 60(6), 1409-1411 (1977).

${ }^{13}$ E. L. Madsen, H. J. Sathoff, and H. J. Zagzebski, "Ultrasonic shear wave properties of soft tissues and tissuelike materials," J. Acoust. Soc. Am. 74(5), 1346-1355 (1983).

${ }^{14}$ X. Yang, R. A. Roy, and R. G. Holt, "Bubble dynamics and size distributions during focused ultrasound insonation," J. Acoust. Soc. Am. 116, 3423-3431 (2004).

${ }^{15}$ J. S. Allen, R. A. Roy, and C. C. Church, "On the role of shear viscosity in mediating inertial cavitation from short-pulse, megahertz-frequency ultrasound,” IEEE Trans. Ultrason. Ferroelectr. Freq. Control 44, 743-751 (1997).

${ }^{16}$ C. C. Church, "Prediction of rectified diffusion during nonlinear bubble pulsations at biomedical frequencies," J. Acoust. Soc. Am. 83, 2210-2217 (1988).

${ }^{17}$ C. K. Holland and R. E. Apfel, "An improved theory for the prediction of microcavitation thresholds," IEEE Trans. Ultrason. Ferroelectr. Freq. Control 36, 204-208 (1989). 